Revue européenne des sciences sociales

European Journal of Social Sciences

XLIV-134 | 2006

Quel(s) défi(s) pour les sciences sociales à l'heure de la mondialisation?

\title{
Réflexions hétérodoxes sur la globalisation
}

\section{Claude Raffestin}

\section{(2) OpenEdition \\ Journals}

Édition électronique

URL : http://journals.openedition.org/ress/308

DOI : $10.4000 /$ ress.308

ISSN : 1663-4446

Éditeur

Librairie Droz

Édition imprimée

Date de publication : 1 juin 2006

Pagination : 247-257

ISBN : 9-782-600-01095-5

ISSN : 0048-8046

Référence électronique

Claude Raffestin, "Réflexions hétérodoxes sur la globalisation », Revue européenne des sciences sociales [En ligne], XLIV-134 | 2006, mis en ligne le 15 octobre 2009, consulté le 19 avril 2019. URL : http://journals.openedition.org/ress/308; DOI : 10.4000/ress.308 


\section{Claude RAFFESTIN}

\section{RÉFLEXIONS HÉTÉRODOXES SUR LA GLOBALISATION}

\section{DES CHOSES AUX MOTS}

Entre l'usage des mots et leur enregistrement dans les dictionnaires, il y a, c'est l'évidence même, un écart temporel pas toujours facile à repérer. Le terme de globalisation, selon le Robert, date de 1968, mais il a été précédé en 1963 par celui de mondialisme, synonyme appartenant à la même constellation sémantique quand bien même avec de sensibles différences. Dans le même ordre d'idée, l'adjectif universaliste à connotation religieuse, de 1684, a donné universalisme qui est relevé en 1823. Il est évident, bien entendu, que ce n'est pas parce qu'il n'y a pas de mot pour désigner une chose que celle-ci n'existe pas.

Le processus de globalisation actuel, essentiellement économique, aurait débuté selon certains durant la troisième décennie du $\mathrm{XIX}^{\mathrm{e}}$ siècle quand on a pu noter une tendance des prix des biens à converger vers un prix unique ${ }^{1}$. Cette tendance, annonciatrice de ce que nous connaissons aujourd'hui, a cependant été précédée par des phénomènes de globalisation qu'il est loisible d'évoquer, même si ceux qui les ont vécus n'en ont pas toujours été conscients. La révolution agricole, la révolution urbaine et la révolution industrielle, pour ne citer que ces exemples, ont été des phénomènes de globalisation qui n'ont pas été qualifiés ainsi, probablement en raison de la «lenteur» de leur diffusion, qui n'a pas permis d'en prendre une conscience suffisante dans l'enveloppe spatio-temporelle. L'étalement dans le temps, différentiel selon le type de révolution, n'a pas permis d'en saisir le caractère globalisant, en raison de l'échelle d'observation, toujours créatrice du phénomène. J'aurai garde d'oublier tous les phénomènes de globalisation liés à la technique, à la science et à la culture dont le rôle a été énorme au XIX siècle, à travers la divulgation et la vulgarisation dont certains auteurs ont été les acteurs privilégiés.

Si Alexander von Humboldt était à nouveau parmi nous, et revenu, bien entendu, de ses premiers étonnements, il ne mettrait probablement pas longtemps à comprendre que ce qu'il avait tenté de faire, donner une vision globale de la terre, s'inscrivait dans un mouvement d'universalisme de la science et de la culture. Il en irait de même pour Elisée Reclus dont l'œuvre s'inscrit dans le même courant. On peut supposer que l'un et l'autre, parfaitement conscients de ce phénomène «globalisant» auraient pu aller jusqu'à l'expliciter, à travers les

A. Vercelli, S. Borghesi, La sostenibilità dello sviluppo globale, Roma, Corocci editore, 2005, p. 17-18. 
principes d'interrelation et d'interaction qu'ils ont, mieux que d'autres, illustrés. Ils y ont probablement pensé, mais sans le faire. D'ailleurs pourquoi l'auraient-ils fait puisque tous les deux étaient absolument conscients de la «globalisation de la connaissance».

\section{FLUX DE CONNAISSANCES ET D'INFORMATIONS}

Dans les phénomènes de globalisation évoqués plus haut, ce qui est à l'œuvre, à l'origine du moins, ce sont toujours des systèmes de connaissances et d'informations. Les connaissances et les informations, liées à l'agriculture, à la ville et à l'industrie, sont progressivement modifiées, rapidement ou lentement. Il est évident, qu'en leur temps, ces phénomènes de globalisation ont apporté des transformations et des bouleversements qui n'ont été compris et intégrés, le plus souvent, qu'avec un certain retard.

Dans tous les phénomènes de globalisation, on se trouve en présence d'une réorganisation des systèmes matériels, bien sûr, mais aussi et surtout immatériels existant à partir de nouvelles combinaisons informationnelles. Les connaissances peuvent n'être pas nouvelles, mais combinées d'une manière nouvelle, qu'il s'agisse d'information fonctionnelle, celle destinée, au sens large, à «produire» ou d'information régulatrice, destinée à éviter les dysfonctionnements dans les diverses structures, pour les conserver en état de fonctionner. Par ailleurs, si dans les sociétés traditionnelles, par opposition aux sociétés modernes, on observe une liaison étroite entre pratiques et connaissances, autrement dit une fusion des unes et des autres; au contraire, dans les sociétés modernes et post-modernes, on note une fission des unes et des autres. Comme l'a sous-entendu Serge Moscovici, l'histoire humaine de la nature a toujours procédé par globalisations successives, l'une se substituant à l'autre, mais en laissant des traces importantes dans l'existence des hommes et dans les lieux qu'ils habitent et ont habités ${ }^{2}$. Lorsqu'il est question d'état de nature organique, d'état de nature mécanique et/ou d'état de nature synthétique ou cybernétique, Moscovici fait allusion à des révolutions/ évolutions dans lesquelles les sociétés ont remanié l'entièreté de leurs relations avec ce qu'il est convenu d'appeler la nature et avec eux-mêmes.

A l'occasion de ces remaniements successifs, les modèles et les théories dont les hommes se sont servis ou si l'on préfère les «images» qui ont orienté leurs actions se sont également modifiées. On notera, parce que c'est d'une très grande importance, que l'écart ou mieux «la distance», entre les représentations que les hommes ont créées et la matérialité des choses, a eu une forte tendance à croître au fil du temps. Au fur et à mesure que l'on est passé d'un état de nature à un autre, le rapport aux signes des choses, au détriment des relations avec la réalité matérielle elle-même, est devenu toujours plus étroit. Il en résulte que la relation aux choses est finalement de plus en plus dissimulée, voire cachée sous les signes qui la représentent. On finit par se mouvoir uniquement dans un monde de signes, au

S. Moscovici, Essai sur l' histoire humaine de la nature, Paris, Flammarion, 1977 (1 $1^{\text {are }}$ édition 
risque de perdre, toujours davantage, la relation avec le réel. On oublie que si on peut manipuler les modèles et les théories ou, si l'on préfère, les «images », on ne peut pas les habiter. Pourquoi? Parce que ces images ne sont rien d'autre que des représentations cohérentes mais déformées, exprimées dans divers types de langages, à une échelle donnée, selon la fonction qu'elles doivent assumer. Je dis langages, au pluriel, car effectivement on peut utiliser divers langages: plastique (dessin, peinture, sculpture, photographie), formel comme le langage logicomathématique ou encore et tout simplement, la langue naturelle. La réalité matérielle ou structure est rythmée par des durées ou temps différentiels. La représentation de cette structure est un instantané qu'on peut qualifier d'image. En jouant sur la variété des langages et des échelles (le sens donné ici au mot est plus large que celui habituellement employé en cartographie), on peut obtenir une multitude d'images différentes qui, par leur nature même, figent le temps. Ce sont donc des documents historiques dont l'écart avec la réalité peut être considérable. Documents historiques, certes, mais aussi «coupes» susceptibles de renseigner sur tel ou tel aspect de la structure.

L'image, en tant que moyen d'accéder à une pratique et à une connaissance du réel, a toutes les caractéristiques de la monnaie fiduciaire qui permet d'obtenir une richesse contre le signe d'une richesse ou le contraire. Ce n'est pas parce que la valeur actuelle d'une représentation est faible que sa valeur future le sera. Bien au contraire, car la valeur d'une représentation sera d'autant plus grande que l'objet représenté sera moins accessible ou même inaccessible dans sa réalité originelle. De la même manière que la monnaie fiduciaire est le signe de la richesse et un moyen d'échanger la richesse et de la faire circuler, la représentation est le signe d'une réalité, le moyen de la connaître et de la faire circuler. Dans ce cas, on peut faire une comparaison homothétique comme le paysage qui est le signe du pays et également un moyen de le faire connaître et de le faire circuler, mais n'est évidemment pas le territoire. Le territoire a commencé à être paysage quand il a commencé à être pensé: «Commencé à être pensé » signifie prendre une distance par rapport à la chose. L'objet est, en quelque sorte, transformé pour être échangé. Etre transformé pour être en situation d'être communiqué. La transformation se fait toujours par le truchement d'un langage et les représentations qui en découlent constituent, comme je l'ai dit plus haut, une monnaie fiduciaire pour avoir accès au réel non immédiatement appréhendable. De la même manière que la monnaie permet de fracturer, dans le sens de fragmenter, la richesse réelle, pour l'échanger; l'image permet de «découper» la réalité matérielle pour la communiquer.

La représentation prend une valeur considérable quand elle réussit à avoir cours légal dans les esprits, à l'intérieur d'une société. Il est particulièrement intéressant de constater que le mécanisme en cause, légèrement paradoxal à certains égards, réside dans le fait que la réalité matérielle ne prend vraiment de l'importance et de la signification que lorsque la représentation a réussi à s'imposer et donc, d'une certaine manière, a réussi à se substituer à elle. Le mécanisme de la monétarisation fiduciaire a alors fonctionné puisque si d'aventure la chose réelle venait à disparaître, cela serait certes catastrophique, mais il y aurait toujours les signes de la représentation pour la suggérer et éventuellement permettre de la recréer. Cette dernière hypothèse est évidemment plus problématique sans pour autant être inconcevable. 


\section{LA GLOBALISATION ACTUELLE}

Le processus de globalisation que nous affrontons aujourd'hui est: «Une révolution technique, centrée sur des procédés informationnels, (qui) remodèle à un rythme accéléré les fondements matériels de la société. Les économies deviennent partout mondialement interdépendantes et introduisent ainsi une nouvelle forme de relation entre l'économie, l'Etat et la société, dans un système à géométrie variable $»^{3}$. Dès les premières pages de son livre, Manuel Castells cherche à expliquer la globalisation en cours, par l'emprise toujours plus grande du capital sur le travail, du déclin du mouvement ouvrier, de l'individualisation et de la diversification des relations de travail. Pour reprendre ce que j'ai avancé plus haut, il est dommage que Castells n'ait pas compris ou voulu comprendre que la globalisation, telle qu'il la définit, est une formidable (mot à prendre au sens étymologique, à savoir «qui inspire la crainte») transformation voire mutation de la relation aux choses, d'une part, et une chosification des relations aux hommes, d'autre part. Les hommes et les choses sont vus à travers le prisme, évidemment déformant, des signes. Cela a toujours été le cas, sans nul doute, encore qu'à des degrés divers, mais ce qui est particulièrement préoccupant, c'est que la combinaison des deux monnaies fiduciaires, à savoir celle des représentations et celle de l'argent, tendent à masquer complètement les réalités matérielles. Le double système d'images se télescope sans cesse. Les choses se passent comme lorsqu'on se demande, le lendemain de la projection d'un film télévisé, quelle est la part de la fiction et quelle est celle de la publicité qui n'a pas cessé de s'intercaler entre les diverses scènes. Le référent, ici le film, est complètement brouillé et la mémoire peut en être perturbée.

A un moment donné, mais presque subrepticement, Castells glisse une phrase qui semble être une clé possible de ce qu'il énonce et tente de décrire: «Entre autres conséquences de cette révision générale du système capitaliste, nous assistons à l'intégration planétaire des marchés financiers...»" La phrase est importante, mais c'est en quelque sorte une incidence. Pour comprendre que ce sont les flux financiers qui importent vraiment, il faut chercher la réponse dans l'ensemble du livre. Castells n'insiste pas, outre mesure, quand il y fait allusion, sur l'importance des signes. Certes, on les retrouve dans le savoir et dans l'information: « Si un accroissement du savoir peut normalement entraîner une augmentation de rendement par unité de production, c'est la recherche de savoir et d'information qui, dans le mode informationnel, caractérise la fonction technologique de production». Cela étant dit: «... le facteur historique qui, de la manière la plus décisive, a accéléré, canalisé et façonné le paradigme de la technologie informationnelle, et les formes sociales qui l'accompagnent, a été et est toujours le processus de restructuration capitaliste entrepris depuis les années 1980, à tel point que le nouveau système techno-économique peut être légitimement qualifié de capitalisme informationnel.» $\gg^{5}$ Dans ces conditions, la technologie informationnelle et le savoir ne sont que des moyens. Plus loin, Castells l'énonce clairement:

\footnotetext{
M. Castells, La société en réseaux. L'ère de l'information, t. 1, Paris, Fayard, 1998, p. 21.

Castells, ibid. p. 22.

Ibid., p. 39-40.
} 
«Les premiers bénéficiaires, et les plus directs, de ces restructurations ont été les acteurs mêmes de la transformation techno-économique: les entreprises à haute technologie et les grandes sociétés financières. ${ }^{6}$ Les flux financiers internationaux ont augmenté dans des proportions énormes par rapport au PIB: «Pour la première fois de l'histoire, le capital se gère jour et nuit sur des marchés financiers opérant en temps réel: des transactions représentant des milliards de dollars s'effectuent en quelques secondes sur les circuits électroniques du monde entier.» ${ }^{7}$

Dans ces conditions, l'élément le plus important et le plus significatif dans les réseaux est «l'argent qui est devenu presque entièrement indépendant de la production, y compris de la production des services, en empruntant des réseaux d'interactions électroniques d'un ordre supérieur que comprennent à peine leurs opérateurs. $\gg^{8}$ Je ne voudrais pas sombrer dans le délire d'une interprétation catastrophiste, mais il convient de se poser des questions quant à l'argent dans le phénomène de globalisation. En tant que substance totalement dématérialisée et fluide, l'argent n'est évidemment pas le but en soi, même si faire de l'argent avec de l'argent est devenu une activité fondamentale. Le pouvoir ne réside pas dans l'argent mais dans l'orientation donnée aux flux informationnels mobilisés par les flux financiers. La disponibilité des signes monétaires et la capacité de faire circuler ceux-ci pour posséder, avant tout le monde, ce qu'on appelle pudiquement un avantage technologique, constitue le levier pour orienter la production de connaissances et d'informations. Aujourd'hui, le travail le plus significatif est celui d'invention et d'innovation que les signes monétaires permettent d'acheter ou de contrôler étroitement. Le monde s'inquiète, je veux dire que les Etats s'inquiètent du contrôle quasiment absolu exercé par les Américains sur Internet. Quoi de plus emblématique que la «toile» pour prendre la mesure de la circulation du double système de signes? Tout est complètement détaché de la réalité, mais personne ne s'en préoccupe, tout peut être faux ou faussé, mais personne ne s'en avise et chacun semble avoir une confiance aveugle. Qui oserait dire, en effet, que le roi est nu, s'il l'est vraiment?

A ce propos, il faut rappeler une série de banalités pour tenter de comprendre ce qui se passe. La connaissance des choses et l'argent, susceptible d'établir des équivalences, ne sont finalement que des «signes». Les connaissances sont d'ailleurs, au même titre que l'argent, une «monnaie fiduciaire» pour faire circuler la réalité, sous la forme d'images qui peuvent être, entre autres, des modèles et des théories. Ces images ont naturellement cours légal, dans la société, du moins pendant une certaine durée. Cette durée est généralement fonction du «temps d'exploitation» capable de dégager des marges bénéficiaires significatives, c'est-à-dire des surplus de signes monétaires.

Lorsque ces images présentent moins d'intérêt, c'est-à-dire que leur cours baisse, les flux d'argent se réorientent dans d'autres directions et déclenchent des processus qui s'appuient sur les structures informationnelles créatrices de nouveaux signes.

Nous sommes maintenant dans une société de signes. Je veux dire que «l'idéel» devient plus significatif que le «matériel» et se substitue à celui-ci

\footnotetext{
Ibid., p. 113.

Ibid., p. 123.

Castells, op. cit., p. 530.
} 
chaque fois que cela est possible. La globalisation postule une société de signes, puisque, pour fonctionner, il s'agit d'établir un système généralisé d'équivalences et de veiller à la plus grande fluidité des signes, compatible avec les ressources du système. Pour réaliser la fluidité la plus grande, il convient d'augmenter la distance entre réalité et signe. Cela n'est possible que si la réalité tend à être «oubliée » ou, si l'on préfère, tend à être «refoulée» et cela n'est possible qu'au prix de la fission entre réalité et signe.

La «réalité » n'aurait-elle plus d'importance? Bien au contraire, puisque comme élément de moins en moins visible, elle devient prétexte à faire du signe sous toutes les formes possibles et imaginables et on assiste à une inflation sémique. Il y a homologie entre inflation monétaire et inflation sémique. L'inflation sémique est, en partie, une conséquence de la nécessité de faire sans cesse de la «nouveauté» pour en dériver, en conséquence, des signes, monétaires. Il y a un rapport étroit entre néophilie et globalisation. Le pouvoir est détenu par ceux-là mêmes qui contrôlent la production, l'échange et la consommation des signes et, par conséquent, on ne cesse pas de produire, d'échanger et de consommer des signes avec des signes. La globalisation peut être définie comme une production de signes avec des signes. Ce ne sont pas n'importe quels signes qui sont en cause, mais bien ceux des choses essentielles et fondamentales qui sont à la base même de l'existence.

\section{LES SIGNES DES «CHOSES ESSENTIELLES»}

Ce sont les signes des grandes logiques. Celles qui ont structuré progressivement la planète et la structurent encore. Elles sont apparues à des moments différents. D'abord l'éco-logique, puis la bio-logique, et enfin, en tout dernier lieu, l'anthropo-logique dont les interactions et les interrelations ont produit notre monde. Ces grands ensembles de «logiques» constituent les fondements essentiels de la réalité matérielle et sont par conséquent ce qui est donné à représenter pour tenter de comprendre le monde: elles sont, à elles seules, des formes de globalisation. Elles structurent nos existences et elles sont essentielles puisqu'elles sont le fondement de la vie. Elles sont menacées, parce qu'elles constituent un enjeu fabuleux. Qui s'en empare, peut intervenir dans nos vies et sur nos vies. Qui les détruit, ne serait-ce même que partiellement, menace nos vies.

C'est pourquoi la représentation de ces grands ensembles de «logiques » a été et demeure l'enjeu, donc l'objectif final des cultures qui se sont succédées à travers l'histoire. Ces cultures ont contribué à construire, à l'origine, des mythes, c'est-à-dire des images que nous appelons non-scientifiques, mais qui sont souvent au départ d'images scientifiques, soit des théories et des modèles qui ne sont rien d'autre, somme toute, que des représentations cohérentes, mais déformées de la réalité, autrement dit des «caricatures». Ces images, mythes ou constructions scientifiques, sont évidemment essentielles pour pouvoir comprendre, expliquer et manipuler le réel. Je sais que l'idée de caricature est péjorativement connotée, mais je l'utilise dans le sens de la mesure que l'observateur prend de la dimension du réel pour la communiquer, compte tenu des ressources conceptuelles à sa disposition et créer, par là même, les conditions d'une relative objectivité. Dans le sens traditionnel, la caricature se réduit à l'élément 
déformé, mais pourtant créateur de synthèse. Il faut le répéter, c'est moins la «ressemblance forcée» que la «cohérence de la déformation» qui peut déclencher l'acceptation et l'adhésion.

Sur le plan scientifique, les informations et les connaissances, accumulées sur les choses essentielles, sont particulièrement connotées par l'historicité. Elles n'ont, par conséquent, qu'une signification limitée dans le temps, même si certaines ont été «valables » pendant des siècles. Beaucoup d'autres, en revanche, ne l'ont été que pendant très peu de temps. L'accumulation d'informations et de connaissances sur les choses essentielles constitue une sorte d'arsenal instrumental qui nous permet d'entretenir des relations avec les signes de la réalité matérielle, mais pas directement avec celle-ci.

Pour le dire encore plus simplement, nous vivons plus dans et par les signes que dans et par les réalités. Combien de fois, dans une journée, avons-nous plus recours à des signes qu'à des choses réelles que nous n'avons jamais vues? Il est inutile de chercher à faire un inventaire, car c'est le cas le plus fréquent. Toute banale que peut paraître cette remarque, elle n'en est pas moins au cœur d'une problématique dangereuse qui transforme tout ce que nous touchons en un double système sémique. De la même manière que le roi Midas a risqué de mourir d'inanition parce que tout ce qu'il touchait se transformait en or; nous risquons de tout détruire par notre manière de tout transformer en signes, ce qui est inévitable, mais en coupant notre relation à la réalité.

C'est justement parce que nous vivons dans les signes et par les signes, auxquels nous ne sommes généralement pas en mesure d'attribuer une probabilité de véracité ou de valeur, que surviennent tous les malentendus possibles, toutes les fausses équivalences et toutes les tromperies imaginables.

En effet, à travers notre manipulation des signes des choses essentielles, sans nous préoccuper de savoir, au moment où nous le faisons, si ces signes véhiculent une partie significative ou non de la réalité qu'ils représentent, nous préparons par nos actions les désastres de demain. Quelle est, en effet, la probabilité que ces signes qui ont cours légal dans nos esprits, constitue une bonne représentation de la réalité matérielle avec laquelle nous sommes en train de jouer? Quelle est, dans ces conditions, la valeur des jugements que nous portons et la pertinence des actions que nous entreprenons à partir de ces jugements?

En d'autres termes, quelle est la probabilité de représentation de la réalité des signes que nous utilisons? Toute l'information utilisée, quotidiennement, est sujette à caution. En d'autres termes, sa probabilité de véracité et sa probabilité de représentabilité sont peu élevées.

Il me revient en mémoire une discussion vieille d'une trentaine d'années, avec un académicien soviétique à propos de l'état de l'environnement en URSS. A entendre ce géographe, dont je tairai le nom, par charité, l'état écologique de son pays était au plus haut niveau possible. On savait, pourtant, déjà suffisamment de choses pour comprendre que la probabilité de véracité de son discours était faible, mais, à l'époque, il était encore difficile de savoir jusqu'à quel point elle l'était. Les données statistiques soviétiques étaient gravement manipulées, dans tous les domaines, pour faire apparaître une situation favorable qui confinait à l'utopie. Même si la situation s'est améliorée depuis, nous sommes encore loin d'une probabilité satisfaisante. Les statisticiens savent bien, par exemple, que les données démographiques, selon les lieux et les moments, sont peu fiables. On aurait tort de 
penser, cependant, que tous les signes produits par les pays occidentaux développés, à propos de certaines réalités essentielles, n'aient pas souffert et ne souffrent pas encore du même syndrome. Certes, les probabilités sont vraisemblablement un peu plus satisfaisantes, mais nul n'est à l'abri de ces «monnaies fiduciaires » dévaluées qui compromettent notre rapport au réel.

On pourrait penser qu'il s'agit de reprendre la vieille question des critères de vérité, pour débattre du vrai et du faux et celle de l'idéologie, à l'œuvre dans tout processus de représentation. La question est de savoir pourquoi l'intervalle, entre le signe qu'on donne à consommer et la réalité qui lui sert de référent, est toujours plus grand? Il faut dire que la distance entre le signe et la réalité peut être «infinie », si l'acteur n'a jamais l'occasion d'avoir accès à celle-là. Or, l'accès à la réalité, contrairement à ce que l'on pourrait penser, est de moins en moins aisé. Il ne s'agit donc ni d'un problème de critère de vérité ni non plus d'un problème idéologique mais de tout autre chose qu'on peut essayer de résumer par la formulation du double rapport suivant:

\section{représentation/réalité matérielle monnaie fiduciaire/richesse réelle}

Notre rapport à la réalité matérielle est en crise, en ce sens que nous la manipulons, sans le savoir et sans être en mesure de juger de l'usage que nous en faisons. Nous ne le sommes pas, parce que l'expérience que nous avons des choses est toujours plus médiatisée par des signes. Si la culture consiste à produire et à manipuler des signes, il convient de ne pas oublier qu'elle est, aussi et peutêtre, d'abord rapport à la matière et à la réalité. Si la culture est production, échange et consommation de signes, c'est parce qu'elle est sous-tendue par la production, l'échange et la consommation d'éléments matériels, immatériels, réels et imaginaires.

C'est tout le problème du référent qui est posé, ici. On sait qu'en linguistique, pour reprendre une vieille définition, «on appelle référent ce à quoi renvoie un signe linguistique, dans la réalité extralinguistique telle qu'elle est découpée par l'expérience d'un groupe humain »'. Or nous avons de moins en moins accès à cette expérience.

Ce phénomène de décollement du signe et du référent est, naturellement, accentué par l'autre partie du rapport qui intéresse la représentation de la richesse réelle par les signes monétaires. L'argent, susceptible d'établir des équivalences entre toutes ces choses, n'est finalement lui-même qu'un système de signes. L'équivalence entre réalité et monnaie tend à faire oublier que celle-ci n'est que le signe de la richesse et en aucune façon la richesse elle-même. Nul ne saurait nier que l'invention de la monnaie a constitué un progrès fantastique, pour la circulation des richesses, mais aujourd'hui, on doit se demander si l'instrument monétaire, devenu de plus en plus abstrait, n'est pas en train de faire perdre tout contact avec le réel ou d'accentuer la perte de contact avec le réel. Il suffit de penser à ces droits de polluer, inventés par l'économie, qu'on peut acheter, négocier et revendre, en dehors de toute référence à la réalité humaine qui la sous-tend !

9 Dictionnaire de linguistique, Paris, Librairie Larousse, 1973, p. 415. 
L'hypothèse que nous formulons est que la globalisation sous-tendue par le double rapport, représentation/réalité matérielle - monnaie fiduciaire/richesse réelle, constitue un mode d'appréhension abstrait qui tient compte, de moins en moins, de la régulation de l'environnement. Pourquoi? Parce que seule une infime partie de la population est en mesure de comprendre ce qu'est l'environnement. De plus en plus, les décisions sont prises sur la base de ce double rapport complètement vidé de toute relation au réel pour la bonne et simple raison que les deux parties de cet ensemble représentation linguistiqueléquivalence monétaire fonctionnent sur une structure de même nature. Elles se réclament d'une structuration semblable qui facilite le «décollement» entre signe et réalité.

Par ce double processus d'emboîtement, les deux systèmes de signes se renforcent l'un l'autre et assurent la fluidité de l'information et celle de la monnaie. Ainsi, est apparemment garantie la cohérence de la globalisation. En effet, à l'échelle du signe, les diversités et les différences des réalités sont partiellement gommées et apparaissent moins nettes: le structurel l'emporte sur le factuel. Elles existent bel et bien, mais à l'échelle d'observation des signes, elles ne sont plus prises en compte. Les grandes logiques, créatrices de diversité et évoquées plus haut, peuvent être, dès lors, abstraitement manipulées à travers des assimilations et des équivalences forcées, à l'occasion desquelles il est toujours plus difficile, voire impossible, de prendre conscience des dommages et des destructions, parfois irréversibles, à l'échelle humaine, subis par les réalités environnementales. A maints égards, il devient difficile, sinon impossible, de connaître «l'état du monde » qui nous parvient sous forme d'images et de modèles qui ont cours légal jusqu'à la prochaine crise qui révélera leur relative inadaptation. A ce moment-là, les images présenteront moins d'intérêt et les flux d'argent s'en détourneront, déclenchant, alors, de nouvelles créations en s'appuyant sur les structures informationnelles pour produire de nouveaux signes.

Dans la globalisation, les signes qui sont créés peuvent l'être sans qu'il soit nécessaire de recourir à un référent. Ce n'est plus la dichotomie entre réalité et signe de la réalité qui fonctionne, car la réalité, le plus souvent, est mise de côté et n'est plus qu'un prétexte à faire du signe. La production de signes finit par masquer les destructions qui affectent les grandes logiques sous-tendant les écosystèmes réels. Il suffit de produire de nouvelles morphologies pour dissimuler la crise des écosystèmes réels car celles-là ne sont pas parcourues par des flux réels nouveaux, mais par ceux-là mêmes qui sont en crise parce qu'insubstituables.

Le phénomène est celui, bien connu en marketing, du produit qui se vend mal: on change l'apparence et on adapte la publicité. Le pouvoir est donc détenu par ceux-là mêmes qui contrôlent l'entièreté du double rapport des deux systèmes de signes.

La question qu'il convient de se poser est de savoir si la manipulation simultanée des deux systèmes de signes permet d'intégrer l'enveloppe spatio-temporelle qui bien évidemment nécessite que l'on prenne en compte non seulement les échelles spatiales, mais encore temporelles. La manipulation n'offre pas la possibilité de tenir compte des deux systèmes d'échelles simultanément. Il n'est, en effet, pas possible d'établir des équivalences entre des éléments réels caractérisés par des durées différentes. La première logique a été seule en exercice à l'origine pendant le $15,5 \%$ du temps de la terre. La deuxième, caractérisée par la vie, a 
occupé $84,4 \%$ du temps de la terre. La troisième, la logique anthropique, est venue s'ajouter, il y a quelques centaines de milliers d'années. Toutes ces logiques agissent en interaction, mais il est évident que les effets de la dernière, malgré leur apparition tardive, ont bouleversé tout le système terre, à une vitesse qu'il est loisible de qualifier de vertigineuse !

Ces trois logiques définissent les conditions de viabilité de l'interface biosphèresociété-environnement. Il apparaît, dès lors, que la globalisation est inscrite, dès l'origine, dans les grandes logiques éco- d'abord, puis bio- ensuite et enfin anthropo. La résultante des deux premières est un champ vital globalisé, avec la dernière c'est la «culturalisation » du champ vital et l'achèvement de la globalisation. Ce qu'il faut retenir c'est que toutes les cultures sont porteuses d'un potentiel de globalisation que fort peu d'entre elles sont en mesure d'actualiser. L'origine de la connaissance est aussi celle de l'ordre, c'est-à-dire de la classification symbolique et, c'est assez dire qu'avant cette découverte, il n'y a pas de véritable globalisation possible, mais c'est dire aussi, en même temps, qu'il n'y a pas qu'une seule globalisation possible, comme celle proposée et fondée sur la convergence de deux systèmes de signes que nous avons évoqués.

Cela dit, il est évident que le système réel des trois logiques offre la possibilité de suivre l'évolution spatio-temporelle de la viabilité et de l'utiliser pour une évaluation économique des avantages ou des dommages induits selon l'augmentation ou la diminution de la viabilité, mais faut-il encore que le double système de représentation et d'évaluation jouisse d'une probabilité élevée de véracité sinon tout le système s'écroule à terme.

C'est même la condition sine qua non, pour orienter les choix économiques et socio-culturels dans la recherche du développement durable et culturellement acceptable tant du point de vue global que du point de vue local. La prise en compte des logiques dans leurs interrelations et dans leurs interactions constitue un préalable essentiel à l'analyse de l'empreinte écologique dont la nécessité s'affirme toujours plus dans notre monde globalisé.

Les grandes logiques nous parviennent sous forme d'images et nous devons prendre garde à ne pas oublier que nous pouvons les détruire, même sans nous en rendre compte, tout simplement parce que nous ne pensons plus les décisions en fonction des réalités matérielles, mais en fonction des signes qui les représentent. Le problème ne consiste plus dans les économies globalisées à ménager les réalités matérielles mais à maximiser les rendements des signes monétaires pour agir toujours plus et mieux sur la production d'information et augmenter le pouvoir d'intervention sur l'ensemble de la planète.

Il est beaucoup plus grave de détruire des richesses matérielles que ne pas bien rentabiliser les signes monétaires. C'est pourtant la solution de la rentabilité qui est préférée. Il faut atteindre au moins $15 \%$ de rendement, même au risque de la destruction des écosystèmes. Tout le monde rêve aux taux de croissance chinois qui sont entre 8 et $9 \%$ en moyenne, mais de combien faut-il les amputer pour tenir compte des destructions. Les catastrophes actuelles, en Chine, mais pas seulement, sont assez éloquentes.

Le développement soutenable n'existe pas dans la globalisation car il s'agit de signes et non de réalités matérielles. L'économie des signes est une économie basée sur l'information fonctionnelle, quasi exclusivement, qui tient peu compte de l'information régulatrice quant à l'environnement dans lequel s'exerce l'activité 
économique. Seule la rentabilité financière est prise en compte à l'exclusion de tout autre préoccupation. N'est-ce pas ce qui se passe aux Etats-Unis avec la production excessive de $\mathrm{CO}_{2}$ par habitant? La croissance ne tient pas compte de la croissance des destructions. C'est sans doute pour cela que la santé n'a pas été particulièrement étudiée dans le cadre de la globalisation.

Lorsque Castells écrit: «Nos sociétés se structurent de plus en plus autour d'une opposition bipolaire entre le Réseau et le Soi », il dit tout à la fois beaucoup et peu. Beaucoup, en ce sens qu'il y a exaltation du réseau dont peu parlaient, il y a 25 ans, en ce sens que le Soi est une bulle de savon qui se forme, éclate et se reforme sans que l'on sache comment ça fonctionne. A maints égards, le Soi est devenu une fonction de la société et des techniques qu'elle a à disposition ${ }^{10}$.

La globalisation est la victoire du double réseau de signes qui donne l'illusion de l'indépendance à l'individu, mais qui l'emprisonne, probablement, plus sûrement que les modèles du passé. Ce qui est préoccupant avec la mondialisation, ce n'est pas tant sa forme «totalisante », mais bien plutôt sa tendance à diminuer l'autonomie des populations car celles-ci vont se retrouver enserrées dans des règles souvent garanties par des organismes internationaux du type OMC, plus attentifs aux puissants qu'aux faibles, auxquelles il sera difficile d'échapper, sinon par des pratiques de «ruse» qui demeurent à inventer. Mais c'est une autre histoire! En effet, si nous n'y prenons pas garde, la globalisation pourrait nous amener à gérer la «destruction globale» plutôt que la richesse globale des fondements mêmes de nos existences.

Professeur honoraire

Institut de géographie

Université de Genève

craffestin@hotmail.com

${ }^{10}$ Castells, op. cit., p. 24-26. 